\title{
Comment repenser le rapport de la rhétorique et de l'argumentation?
}

How should we consider the relationship between rhetoric and argumentation?

\section{Michel Meyer}

\section{OpenEdition}

1 Journals

\section{Édition électronique}

URL : http://journals.openedition.org/aad/211

DOI : 10.4000/aad.211

ISSN : $1565-8961$

Éditeur

Université de Tel-Aviv

Référence électronique

Michel Meyer, «Comment repenser le rapport de la rhétorique et de l'argumentation ? ", Argumentation et Analyse du Discours [En ligne], 2 | 2009, mis en ligne le 01 avril 2009, consulté le 23 septembre 2019. URL : http://journals.openedition.org/aad/211 ; DOI : 10.4000/aad.211

Ce document a été généré automatiquement le 23 septembre 2019.

\section{cc) $(1) \ominus$}

Argumentation \& analyse du discours est mis à disposition selon les termes de la licence Creative Commons Attribution - Pas d'Utilisation Commerciale - Pas de Modification 4.0 International. 


\title{
Comment repenser le rapport de la rhétorique et de l'argumentation?
}

How should we consider the relationship between rhetoric and argumentation?

\author{
Michel Meyer
}

1 On distingue généralement la joute dialectique, où s'opposent des thèses adverses, de l'argumentation, qui propose de nouvelles réponses sans forcément entamer de débat contradictoire. On donne simplement des arguments en faveur de la thèse qu'on met en avant. Ces deux formes d'argumentation sont elles-mêmes différentes de la rhétorique. Par celle-ci, il faut entendre à la fois une discipline et des procédés discursifs. La rhétorique comme discipline se démarque de la rhétorique comme ensemble de procédés destinés à plaire et émouvoir. Comme discipline, elle englobe l'argumentation; comme procédé, elle s'y oppose. Aristote parlait d'antistrophos, de pendant à l'argumentation, mais le terme reste imprécis. En quoi la rhétorique est-elle le pendant de l'argumentation? Qu'est-ce que cela peut bien vouloir dire au juste?

2 Il est temps de mettre un peu d'ordre dans toutes ces notions, et pour y parvenir, il faut s'appuyer sur une théorie générale qui remette chaque chose à sa place et les articule de façon cohérente et systématique. La seule théorie de cette nature qui existe depuis l'ontologie aristotélicienne est la problématologie. C'est sur elle qu'on va donc se fonder. Elle offre d'ailleurs une vision assez complète de la raison, du discours, du langage, de l'émotionnel, et même de la science, toutes notions dont la rhétorique fait usage.

3 La rhétorique ne peut se passer du questionnement car, comme le soulignait déjà Aristote, on ne débat que de ce qui fait problème et qui offre des alternatives. En science, la conclusion est certaine, en rhétorique, elle n'est que probable, d'où les alternatives (de réponse), c'est-à-dire des questions et des problèmes. Au fond, si depuis les Grecs, la rhétorique joue un rôle si important dans la pensée occidentale, c'est parce que les réponses s'affaiblissent avec l'Histoire qui s'accélère et qu'elles en deviennent plus problématiques. Ce qui n'est pas sans poser des difficultés. En effet, à côté des réponses problématiques, il y a celles qui restent valables ou qui le deviennent, et il faut arriver à les différencier. La manipulation sophistique repose sur le fait qu'on 
peut faire passer une réponse caduque pour une qui ne l'est pas. C'est de la rhétorique, au sens négatif il est vrai, tel que l'entend Platon dans sa critique des Sophistes. Et puis, il y a l'argumentation, qui se propose de trier les réponses des assertions qui n'en ont plus que l'apparence, étant entendu qu'une vraie réponse, voire une réponse vraie, ne peut l'être que si elle se justifie par une autre déjà validée et qui en est la raison. Comme on le voit clairement, la rhétorique est une discipline qui naît des bouleversements et des confusions qu'occasionne l'Histoire quand elle donne lieu à des amalgames entre le problématique et le non-problématique, affaiblissant les réponses tenues pour telles jusque là, au regard de celles qui vont s'imposer désormais. Argumenter, c'est pouvoir faire un tri par la Raison, par des raisons, de même que les rhétoriser, c'est s'interdire de le faire, en retardant en quelque sorte l'échéance du choix. D'où l'idée que la rhétorique est souvent pur verbiage, de la gymnastique verbale, du vide ou de la flagornerie bien tournée, qui sert à prendre l'auditoire au piège des mots. ("C'est de la pure rhétorique !», comme on entend souvent dire les esprits critiques, lorsqu'ils qualifient ce genre de situations.)

4 La rhétorique et l'argumentation sont donc des modes bien précis pour traiter de certaines questions, et surtout pour évaluer les réponses à disposition, et qui peuvent n'avoir que l'apparence de réponses, pour bien les distinguer de celles qui le sont effectivement, alors même qu'il peut y avoir encore débat. Au fond, si on y regarde bien, il n'y a que deux façons de procéder : soit on avale ces questions en en offrant la réponse, et peut-être en prétendant l'avoir donnée, par l'élégance d'un style bien tourné à cet effet ; soit on les traite comme telles, en les prenant à bras le corps, comme au tribunal où il faut exposer le pour et le contre selon des procédures bien établies, c'est-à-dire équitables. Voilà ce que veut dire le mot antistrophos: la rhétorique est l'envers de l'argumentation, parce qu'on part des questions (rhétorique des conflits) qui opposent des individus, ou des réponses qui les évacuent pour faire croire, à tort ou à raison, que par là elles sont enfin résolues. Ce sont en fin de compte les deux seules manières, distinctes, mais complémentaires, d'attaquer un problème. Bien souvent, quand on ne peut prétendre à la réponse, il faut bien prendre le problème à bras le corps. La rhétorique comme procédé résout le problématique en faisant «comme si » les questions qui l'expriment ne se posaient plus.

D'où le recours à la fiction (symbolisée ici par le « comme si »), au style, aux figures, au discours plaisant, qui doit se substituer à un quelconque argument. On le voit bien dans la publicité, et pas seulement en littérature, pourtant le lieu privilégié de la rhétorique depuis plusieurs siècles. Quand Chanel $n^{\circ} 5$ veut vendre une odeur par l'image publicitaire, la seule façon de procéder est d'appliquer la définition de la rhétorique à la lettre : avaler les problèmes en guise de solution. Si on utilise ce parfum, plus aucun problème ne se posera. Comme c'est impossible, ce ne peut être que magique. Donc, il faut mettre en scène un conte de fées, où tous les problèmes seront résolus comme par enchantement. Les loups ne mangeront plus le Chaperon Rouge, qui les emmènera à la conquête de Paris. Une illusion qui relève de la magie, la magie de Chanel $n^{\circ} 5$, qui envoûte les ennemis et aplanit les difficultés. Il n'y a plus de problème : le petit Chaperon Rouge, parce que " chanélisé », domine la situation.

6 Les problèmes qu'on ne peut ni ne veut évacuer (la lessive, moins enthousiasmante, ne peut pas ne pas mentionner ce qu'elle permet de résoudre) doivent, eux, être argumentés. Une autre logique est nécessaire. Argumenter, c'est donner des raisons, en ramenant le problématique, auquel on est confronté, à du non-problématique, qui lui 
est plus ou moins éloigné, identique. D'où le rôle de l'analogie, de la réduction, mais aussi de l'opposition à ce qui n'est pas acceptable, pour renforcer un état de choses qu'on estime non problématique et qui commande tout le reste.

Rhétorique et argumentation renvoient toutes deux à une question qui mesure tout ce qui sépare (ou réunit, le temps de la poser, ou plus longtemps si la relation est davantage caractérisée par le psychologique) les protagonistes de la relation rhétorique. Ils ne se connaissent pas forcément, comme l'homme politique qui s'adresse par l'intermédiaire de la télévision à son électorat, ou l'écrivain qui ignore quels seront ses lecteurs. La rhétorique, envisagée cette fois comme discipline, réunit toujours un orateur et son auditoire sur une question donnée qu'ils négocient. A travers elle, c'est la distance, la différence entre eux, qu'ils mettent sur la table. Qu'estce qui est hors-question, qu'est-ce qui est en question, qu'est-ce qui, dans leurs positions respectives, est négociable ? Ce sont là les questions de base de la rhétorique (et de l'argumentation). Pour mettre des limites, le militaire porte un uniforme, l'homme d'Eglise une soutane, le patron un costume sobre, etc. Le statut est visualisable ou affirmé, mais toujours identifiable. On négocie souvent ce que l'on est au travers de ce que l'on dit (principe d'adhérence), ce qui fait que l'on vexe souvent des gens avec qui on n'est simplement pas d'accord. Ils se sentent remis en question, précisément du fait des questions qu'on soulève, parfois même en toute innocence. C'est pour cette raison que la relation rhétorique avale souvent ces questions par un discours convenu, voire même flatteur. L'autre ne se sent pas mis en question par de tels discours, dont la fonction n'est pas de dire quelque chose mais de conforter quelqu'un. Celui-ci peut ne pas se l'avouer, et il tombe alors dans ce que j'appelle l'illusion rhétorique, qui consiste à croire que c'est du problème qu'on argumente, alors qu'en réalité, c'est de la position relative des intervenants. On négocie en réalité une distance, une différence (qu'on minimise), alors qu'on semble débattre seulement d'une question, forcément plus objective en apparence car extérieure, par nature, aux individualités.

8 La rhétorique porte donc sur le soi (ethos), sur une question (logos), donc la réponse à y apporter, et sur les autres, c'est-à-dire l'auditoire (pathos). Il est normal qu'on trouve là les trois grands lieux du répondre rhétorique et des arguments invocables. La distance s'annule dans la réponse commune, une distance qui est ponctuelle autant que la réponse. Mais l'accord fait du bien, il donne l'impression aux individus d'être approuvés. La plupart du temps, la rhétorique ne vise pas ce genre d'illusion provisoire et ponctuelle. Elle est sociale: on négocie la distance, les différences, ses réponses propres comme réponses en tant qu'elles nous concernent intimement et socialement. On négocie son Moi, une question, mais aussi sa relation à autrui, d'où le réservoir de réponses, parfois toutes faites, qu'on trouve également dans l'ethos et le pathos. La distance avec autrui peut être simplement négociée, ses effets négatifs annulés, sans être abolie pour autant. Les valeurs, on ne le répétera jamais assez, sont les émotions débarrassées de leur aspect subjectif (on appelle cela des principes). De même, les émotions et les passions sont les valeurs traduites dans le langage de la subjectivité. Jouer sur les valeurs, c'est réduire l'émotionnel dans la négociation intersubjective. $\mathrm{Si}$ la distance est faible, l'émotionnel prend une part plus large dans le débat, car les protagonistes sont proches, donc leurs différends éventuels, plus passionnels. Par contre, si la distance est grande, le recours aux valeurs va être préféré par l'orateur, plus soucieux de garder une certaine objectivité. De toute façon, les trois grandes problématiques, soi, le monde et autrui, sont impliquées à un titre ou à un autre dans la 
relation rhétorique, en même temps qu'elles représentent les lieux où viennent s'enraciner les arguments (leur répertoire, en somme).

9 Articuler tout ceci n'est pas chose facile, ce qui va à l'encontre de l'idée qu'on se fait généralement de la rhétorique, toujours jugée trop « plate » ou même triviale. La prose de Monsieur Jourdain en somme. C'est là une profonde erreur de jugement, et je ne puis que renvoyer ici le lecteur aux analyses détaillées de mes Principia Rhetorica (Paris : Fayard, 2008).

\section{BIBLIOGRAPHIE}

Meyer, Michel. 1994. Rhetoric, Language and Reason (University Park: Penn State University Press)

Meyer, Michel. 2004. La Rhétorique (Paris : PUF)

Meyer, Michel. 2008 [1986]. De la Problématologie (Paris : PUF)

Meyer, Michel. 2008. Principia Rhetorica (Paris : Fayard)

\section{RÉSUMÉS}

La rhétorique, dit Aristote, est le pendant de la dialectique et de l'argumentation. Cela pose le problème de leur harmonisation au sein d'une théorie unifiée, où la rhétorique littéraire voisine avec la logique juridique. La problématologie est cette conception unifiée. Les questions expresses relèvent du conflit argumenté, comme en droit, qui les codifie, et les questions indirectes, des réponses qui les avalent par l'élégance et le style pour se faire passer pour résolutoires de ces questions. La rhétorique est la négociation de la distance entre les individus sur une question donnée, une question plus ou moins problématique et conflictuelle. La problématologie est à la base d'une véritable nouvelle rhétorique, avec de nouvelles prémisses fondées sur le questionnement, laissées jusque-là en friche. Des figures de rhétorique à l'inférence du vraisemblable, le questionnement est le socle où viennent s'articuler la raison, le langage et la persuasion.

According to Aristotle, rhetoric is the counterpart of argumentation. How can we understand the relationship between literary, rhetoric and legal reasoning, if we do not have a general theory of rhetoric? Problematology purports to be precisely that. Questions can be addressed either directly, as in law and legal conflict, where the questions, the pros and the contras are, so to speak, on the table, or indirectly,through their answers, as if they were solved thereby. Rhetoric is the negotiation of the differences between the orator and the audience on a given question, which can be more or less problematic, rhetorical or argumentative. Problematology is the founding theory behind this "new" rhetoric. From the figures of speech to plausible inference, the theory of questioning offers an integrated view of reason, language and persuasion. 
INDEX

Mots-clés : distance psychologique, distance sociale, émotion, problématologie, propositionnalisme, questionnement, valeur

Keywords : emotion, problematology, propositionalism, psychological distance, questioning, social distance, value

\section{AUTEUR}

MICHEL MEYER

Université Libre de Bruxelles 